\title{
Commentary
}

\section{Allosteric Antipsychotics: M4 Muscarinic Potentiators as Novel Treatments for Schizophrenia}

\author{
Martilias Farrell' and Bryan L Roth*,' \\ 'Division of Medicinal Chemistry, Department of Pharmacology, NIMH Psychoactive Drug Screening Program, University of North Carolina \\ Chapel Hill Medical School, Chapel Hill, NC, USA
}

Neuropsychopharmacology (2010) 35, 85 I-852; doi:I 0.I 038/npp.2009.206

The cholinergic system has been implicated in both the etiology and the treatment of schizophrenia for nearly 60 years (Rowntree et al, 1950). With regard to schizophrenia therapeutics, pioneering studies have shown a high correlation between the antimuscarinic properties of typical and atypical antipsychotic drugs and lack of extrapyramidal side effects (EPS) (Snyder et al, 1974); this finding led to the anticholinergic hypothesis of atypical antipsychotic drug action. However, it is now clear that antimuscarinic receptor activity cannot be the sole contributor to the lack of EPS as several atypical antipsychotic drugs exist, which have negligible affinities for all five human muscarinic receptors and low EPS (Roth et al, 2004). A chance observation that xanomeline (a pan-muscarinic receptor agonist with substantial off-target actions at other biogenic amine receptors; Figure 1) diminished psychotic symptoms in patients with Alzheimer's disease (Bodick et al, 1997) led to the proposal that the modest antipsychotic effects of xanomeline might be attributed to M1/M4 receptor agonism (Shannon et al, 2000). Similarly, the pan-muscarinic agonist properties of $\mathrm{N}$-desmethyl-clozapine (Davies et al, 2005) provided additional support for the hypothesis that muscarinic receptor agonism might be antipsychotic (Figure 1).

A major impediment to testing the 'muscarinic agonism' hypothesis of antipsychotic drug therapeutics has been the fact that broad-spectrum muscarinic agonists frequently have debilitating side effects (eg, excessive sweating, piloerection, nausea, vomiting, seizures). Given the prominent roles of M1 and M4 muscarinic receptors in the regulation of dopaminergic neurotransmission (Gerber et al, 2001; Gomeza et al, 1999), M1 and/or M4 selective agonists have been postulated to be potentially safe and effective

\footnotetext{
*Correspondence: Dr BL Roth, Division of Medicinal Chemistry, Department of Pharmacology, NIMH Psychoactive Drug Screening Program, University of North Carolina Chapel Hill Medical School, 8032 Burnett Womack Bldg, Chapel Hill, NC 27759, USA,

Tel: + | 9199667535 , Fax: + | 9198435788 ,

E-mail: bryan_roth@med.unc.edu
}

antipsychotics, although their development has been an elusive goal.

One recent approach for creating drugs, which are highly selective for previously intractable targets, has been to create allosteric modulators, which can either potentiate or inhibit the activity of the receptor (see Christopoulos (2002) for a review). In this issue of Neuropsychopharmacology, a team led by Arthur Christopolous and Patrick Sexton at the University of Monash in collaboration with Eli Lilly report on the molecular mechanisms and potential antipsychotic actions of a novel M4 allosteric potentiator LY2033298.

A recent report by this group (Chan et al, 2008) provided the tantalizing preliminary findings that LY2033298 had antipsychotic actions in various rodent models. Although these findings were interesting, the molecular and cellular mechanism(s) of action of LY2033298 were unclear. Indeed, a key unanswered question from the initial report of Chan et al (2008) was whether LY2033298 was a bona fide allosteric potentiator.

The sine qua non for allosteric potentiation of agonist activity is the demonstration that the drug enhances agonist-binding potency at the receptor. In a series of carefully controlled and quantitatively analyzed studies, Leach et al (2010) showed that LY2033298 potentiates the agonist potency of acetylcholine in both transfected cells and neuronal cell lines, which endogenously express M4 receptors. One of the strengths of this approach is the rigorous quantitative analysis of various parameters associated with allosterism. Indeed, the group of Sexton and Christopolous are major contributors to the current renaissance of quantitative molecular pharmacology.

Leach et al (2010) then showed that LY2033298 potentiates the effect of oxotremorine (a nonselective muscarinic agonist) in the conditioned avoidance response paradigm in WT mice, whereas this potentiation was attenuated but not abolished in M4 KO mice. These findings indicate that in addition to M4 potentiation, there may be a non-M4 component to the activity of LY2033298. The study by Leach et al (2010) stands as a paradigm for the molecular pharmacological characterization of drug-like compounds, 


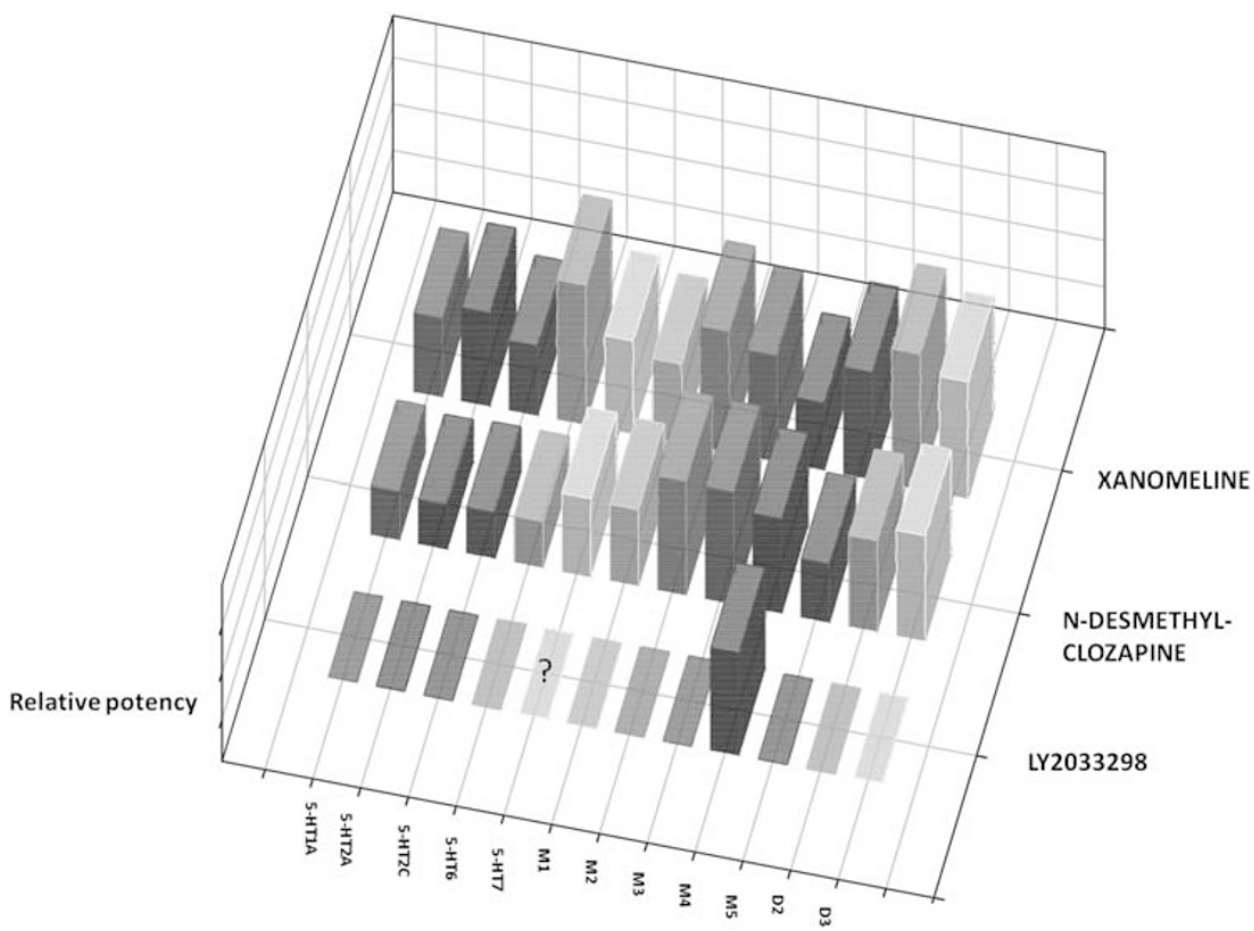

Figure I GPCR profiles of 'selective' muscarinic agonists. The relative comparative activities of xanomeline, N-desmethylclozapine, and LY2033298 at GPCRs implicated in atypical antipsychotic drug actions are shown. As can be seen, only LY2033298 has significant selectivity for muscarinic and nonmuscarinic GPCRs implicated in antipsychotic drug actions. '?' represents unknown activity at 5-HT7 receptors.

which therapeutically modulate GPCR signaling. However, the potential antipsychotic activity of this compound must be considered 'putative' until it can be validated in other models (eg, primates) and tested in humans.

\section{DISCLOSURE}

BLR has been a consultant for the following entities for the past 36 months: Michael J Fox Foundation, National Institute of Health, BMS-Otsuka, ArYX Pharmaceuticals, GlaxoSmithKline, AMRI Inc., Supernus Pharmaceuticals, Epix Pharmaceuticals, Merck, Wyeth-Solvay Alliance, DaiNippon Sumitomo, Invitrogen, LaboPharma, and Medivation. BLR receives grant and contract support from the National Institute of Mental Health and the National Institute of Drug Abuse. MF has nothing to disclose.

\section{REFERENCES}

Bodick NC, Offen WW, Levey AI, Cutler NR, Gauthier SG, Satlin A et al (1997). Effects of xanomeline, a selective muscarinic receptor agonist, on cognitive function and behavioral symptoms in Alzheimer's disease. Arch Neurol 54: 465-473.

Chan WY, McKinzie DL, Bose S, Mitchell SN, Witkin JM, Thompson RC et al (2008). Allosteric modulation of the muscarinic M4 receptor as an approach to treating schizophrenia. Proc Natl Acad Sci USA 105: 10978-10983.

Christopoulos A (2002). Allosteric binding sites on cell-surface receptors: novel targets for drug discovery. Nat Rev Drug Discov 1: 198-210.
Davies MA, Compton-Toth BA, Hufeisen SJ, Meltzer HY, Roth BL (2005). The highly efficacious actions of N-desmethylclozapine at muscarinic receptors are unique and not a common property of either typical or atypical antipsychotic drugs: is M1 agonism a pre-requisite for mimicking clozapine's actions? Psychopharmacology (Berl) 178: 451-460.

Gerber DJ, Sotnikova TD, Gainetdinov RR, Huang SY, Caron MG, Tonegawa S (2001). Hyperactivity, elevated dopaminergic transmission, and response to amphetamine in M1 muscarinic acetylcholine receptor-deficient mice. Proc Natl Acad Sci USA 98: 15312-15317.

Gomeza J, Zhang L, Kostenis E, Felder C, Bymaster F, Brodkin J et al (1999). Enhancement of D1 dopamine receptor-mediated locomotor stimulation in $\mathrm{M}(4)$ muscarinic acetylcholine receptor knockout mice. Proc Natl Acad Sci USA 96: 10483-10488.

Leach K, Loiacono RE, Felder CC, McKinzie DL, Mogg A, Shaw DB et al (2010). Molecular mechanisms of action and in vivo validation of an M4 muscarinic acetylcholine receptor allosteric modulator with potential antipsychotic properties. Neuropsychopharmacology 35: 855-869.

Roth BL, Sheffler DJ, Kroeze WK (2004). Magic shotguns versus magic bullets: selectively non-selective drugs for mood disorders and schizophrenia. Nat Rev Drug Discov 3: 353-359.

Rowntree DW, Nevin S, Wilson A (1950). The effects of diisopropylfluorophosphate in schizophrenia and manic-depressive psychosis. J Neurol Neurosurg 13: 47-62.

Shannon HE, Rasmussen K, Bymaster FP, Hart JC, Peters SC, Swedberg MD et al (2000). Xanomeline, an M(1)/M(4) preferring muscarinic cholinergic receptor agonist, produces antipsychoticlike activity in rats and mice. Schizophr Res 42: 249-259.

Snyder S, Greenberg D, Yamamura HI (1974). Antischizophrenic drugs and brain cholinergic receptors affinity for muscarinic sites predicts extrapyramidal effects. Arch Gen Psychiatry 31: 58-61. 